\title{
Comparison of Mutual Coupling Phenomena in Subwavelength Ridged Circular Apertures and Half-Wavelength Dipole Antenna Arrays
}

\author{
Jong-Eon Park, Ki Young Kim, and Jae-Won Song \\ School of Electrical Engineering and Computer Science, Kyungpook National University, Daegu 702-701, Republic of Korea \\ Correspondence should be addressed to Jong-Eon Park, parkje00@naver.com
}

Received 18 July 2012; Accepted 1 December 2012

Academic Editor: Zhongxiang Q. Shen

Copyright (c) 2012 Jong-Eon Park et al. This is an open access article distributed under the Creative Commons Attribution License, which permits unrestricted use, distribution, and reproduction in any medium, provided the original work is properly cited.

\begin{abstract}
The resonant microwave transmission characteristics of several coupled subwavelength ridged circular aperture arrangements in a thin metallic film are investigated using the three-dimensional finite-difference time-domain (3D FDTD) method. Simple equivalent circuits represented by the self and mutual conductances that have been quantitatively extracted for each resonance condition, including the mutual coupling effects, are also devised with the help of virtual magnetic current elements. Furthermore, a duality is identified between the ridged circular apertures and conventional half-wavelength dipole arrays based on comparing the respective resonance conditions.
\end{abstract}

\section{Introduction}

Bethe's theory of the diffraction of plane electromagnetic waves during transmission through a circular hole in an infinitesimally thin perfect conducting screen states that the power transmitted through the subwavelength hole is very restricted, being proportional to $(a / \lambda)^{4}[1]$, where $a$ is the radius of the circular hole, and $\lambda$ is the wavelength. Moreover, the transmission efficiency is exponentially decreased when the thickness of the screen is considered [2]. As such, this poor transmission efficiency has been perceived as a fundamental restriction in dealing with electromagnetic transmission through a small hole. Therefore, several cousin structures have been studied to overcome this limitation. Harrington analyzed the concept of an electrically small aperture in a conducting screen backed by a conducting body [3], referred to as aperture-body resonance (ABR), where a subwavelength hole loaded with a nearby conductor considerably increases the power throughput when compared with a hole without a nearby scatterer. Inspired by ABR, Leviatan investigated the electromagnetic coupling phenomena between two half-space regions separated by two slot-perforated parallel conducting planes [4]. In this case, when varying the distance between the centers of each slot, a resonant transmission phenomenon is observed, occurring at resonance spacings that approach multiples of $\lambda / 2$. Similarly, the problem of electromagnetic transmission through two slits in parallel plates can be solved with the help of equivalent circuit representation [5]. Here, when the gap between the two plates is close enough, meaning much less than the wavelength of the incident wave, the resulting maximum transmitted power becomes constant, that is, $\lambda / \pi$, irrespective of the slit width and incident angle. Meanwhile, in the case of a thick conducting screen, the resonance transmission has been investigated by altering the thickness of the conducting screen [6]. In this situation, different resonance phenomena are observed when the resonance cavity is built along the longitudinal direction inside the slot region. Alternatively, the addition of two ridges in an electrically small circular aperture also considerably enhances the transmitted power [7-9]. The resonance power transmission of ridged circular apertures as a function of the distance between them was also recently explored [10], experimentally verified [11], compared with dipole arrangements [12], and investigated up to its infinite periodic structure [13]. Furthermore, the recent interest in surface 
plasmons (SPs), followed by Ebbesen's seminal discovery of extraordinary optical transmission (EOT) [14], have led to optical transmission via bowtie, $\mathrm{H}$-, and $\mathrm{C}$-shaped apertures perforated in metallic plates [15-21]. This resonance mechanism is also very similar to the ridged circular apertures used in the microwave region. Extensive analyses of ridged circular apertures (or $\mathrm{H}$-shaped apertures) have already been performed. Here, the resonant frequency of a ridged circular aperture can be adjusted by modifying the width of each ridge and the gap between the ridges without rescaling the host circular shape, while the resonant frequency of a slot antenna, which is one of the basic aperture antenna structures, depends on the width of the slot. Notwithstanding, if the thickness of the metal in the optical regime is considered, the resonant transmission mechanism is not exactly the same for a perfect electric conductor in the microwave region.

However, despite the extensive previous work on ridged structures, an in-depth study of multiple subwavelength resonant apertures is still required and fascinating as well. Once the structure of a single ridged aperture is determined, the arrangement of the multiple apertures becomes an important issue in terms of the potential manipulation of the electromagnetic waves, meaning that the transmission and/or reflection at a particular spectrum of interest can be tailored. Accordingly, this study checks the transmission as a function of the center-to-center distance for vertically and horizontally arranged structures. Inspired by our previous observations [12], the distances for both the minimum and maximum transmitted power are examined for different arrangements and then compared with the spacing distances in conventional dipole arrays. Moreover, as discussed in [12], an equivalent circuit representation is obtained, which is helpful to understand the resonant transmission mechanism. Furthermore, the radiation conductance variation and susceptance variation of a single circular aperture are discussed in the case of a ridge-loaded and ridge-removed aperture. As a result, the self-conductance and mutual conductances for both the maximum and minimum transmitted power in the case of the ridged circular apertures are found to be very similar to the self-resistance and mutual resistances for both the maximum and minimum gains of dipole antenna arrays, allowing the establishment of a duality between them. The usage of a resonant ridged aperture has already been proposed in many applications, including nanoscale lithography [22], single-molecule fluorescence measurement [23], and optical data storage [24-27], and so on [28-30], and this study may be a stepping stone from the single resonant aperture to two-dimensional array of the aperture, for example, a frequency selective surface (FSS) [31], a significant electromagnetic structure with many potential applications.

\section{Resonant Transmission for Various Coupling Cases}

This study presents the problem of electromagnetic wave scattering using subwavelength ridged circular apertures perforated in an infinitesimally thin metallic screen (here,

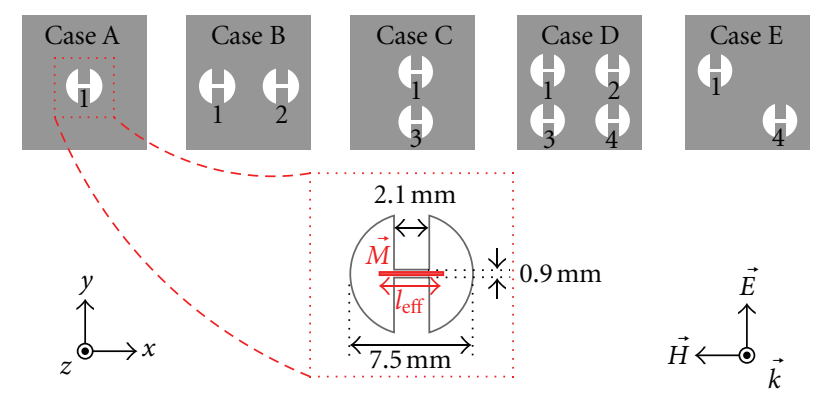

FIGURE 1: Ridged circular apertures perforated in an infinitesimally thin metal screen; (case A: single aperture; case B: collinear; case C: side-by-side; case D: 2-by-2; case E: parallel-in-echelon).

a perfect electric conductor). Five different aperture arrangements, shown in Figure 1, are considered to investigate the mutual coupling phenomena and related physics. The aperture dimensions were arbitrarily chosen to allow the structures to resonate in the X-band $(8-12 \mathrm{GHz})$; the diameter of each circular aperture was $7.5 \mathrm{~mm}$, the ridge width was $2.1 \mathrm{~mm}$, and the gap between the two ridges was $0.9 \mathrm{~mm}$. The plane wave was assumed to be normally incident to the screen from the back side, that is, along the $+z$-direction, while the electric field was linearly polarized along the $y$-direction to cross the ridge. If the polarization of the electric field had been aligned with the ridge, that is, in the $x$-direction, this would have restricted the electromagnetic wave transmission [7]. The three-dimensional finitedifference time-domain (3D FDTD) method [32] was used to analyze the structures. Perfectly matched layer absorbing boundary conditions (PML ABCs) were set up at all six $( \pm x$, $\pm y$, and $\pm z$ ) surrounding boundaries. The structure was numerically solved using a simulation volume of $16.8 \mathrm{~mm} \times$ $16.8 \mathrm{~mm} \times 9.9 \mathrm{~mm}(x-, y$-, and $z$-axes in order $)$ including the PML region. Plus, 25 air buffer cells were used for both the incident and transmitted regions in the $z$-axis. The unit time step for each update of the Maxwell equations was set at $2.60 \times 10^{-13} \mathrm{sec}$, which was determined using the stability criteria and Courant factor of the FDTD algorithm.

From the numerical analysis of the FDTD simulation, a transmission cross-section (TCS) was obtained to calculate the transmission efficiency of the small ridged circular apertures. The definition of a TCS is the ratio of the transmitted power $P_{\text {trans }}$ to the incident power density $P_{\text {inc }}$ as follows:

$$
\operatorname{TCS}\left[\mathrm{m}^{2}\right]=\frac{P_{\text {trans }}[\mathrm{W}]}{P_{\text {inc }}\left[\mathrm{W} / \mathrm{m}^{2}\right]}=\frac{0.5 \times \operatorname{Re}\left[\iint\left\{\vec{E}_{t} \times \vec{H}_{t}^{*}\right\} \cdot d \vec{s}\right]}{\left|\vec{E}_{\mathrm{inc}}\right|^{2} /\left(2 \eta_{0}\right)},
$$

where $\vec{E}_{t}$ and $\vec{H}_{t}$ are the transverse electric and magnetic fields of the aperture, respectively, the asterisk denotes the complex conjugate, and $d \vec{s}$ is the unit cell area of the aperture. The incident power density is given by $P_{\text {inc }}=$ $\left|\vec{E}_{\text {inc }}\right|^{2} / 2 \eta_{0}$, where $\vec{E}_{\text {inc }}$ is the electric field of the incident plane wave with a Gaussian form, and $\eta_{0}(=120 \pi)$ is the intrinsic impedance of the free space. Using this definition, 


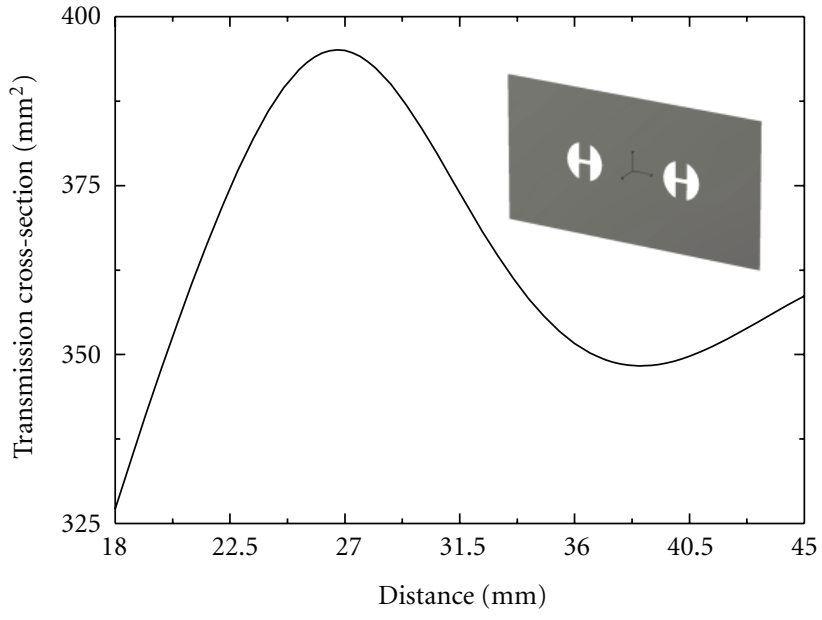

(a)

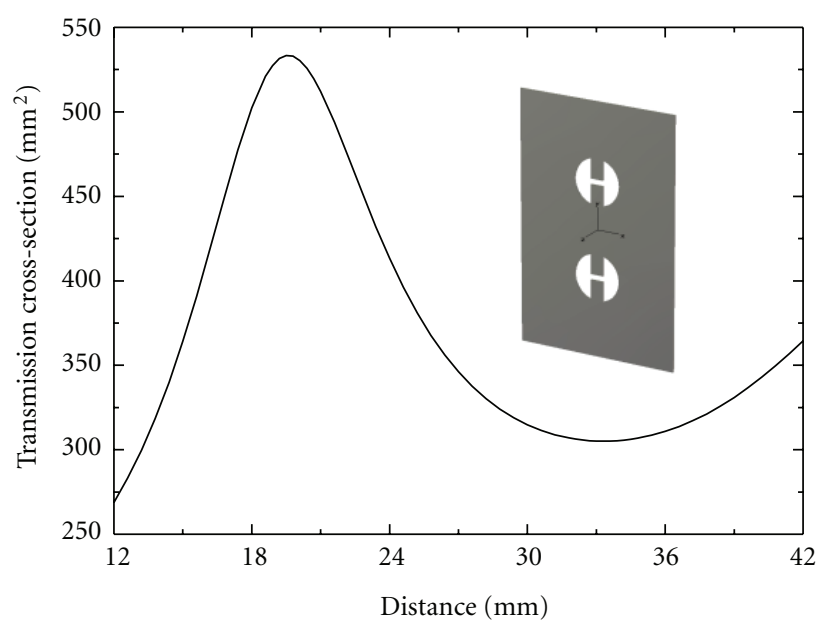

(b)

Figure 2: TCS versus center-to-center distance for (a) case B (collinear case) and (b) case C (side-by-side case).

the TCS for the single ridged circular aperture, shown as case A in Figure 1, was determined as $180.84 \mathrm{~mm}^{2}$ at the resonant frequency of $11.02 \mathrm{GHz}$.

Prior to investigating the TCS variations for the multiple aperture cases, the virtual magnetic current was defined. When the plane wave was incident on the aperture, the $y$ component of the electric field $E_{y}$ was concentrated on the gap between the two ridges (ignoring a small amount of $E_{x}$ ), thereby producing an equivalent magnetic current of $\vec{M}=\vec{E} \times \vec{n}$, depicted as the short red line in Figure 1 . Here, $\vec{E}$ is the induced electric field in the gap between the ridges, and $\vec{n}$ is the normal vector on the $x y$ plane. As will be examined later, the physics of a half-wavelength dipole array also apply to arrays of an equivalent magnetic current $\vec{M}$, which is useful for understanding the coupling mechanisms in coupled ridged circular aperture structures [12]. The classification of the five cases in Figure 1 is based on the arrangement of the equivalent magnetic current elements $\vec{M}$, allowing a convenient comparison of the duality of the current structures and dipole arrays.

Figure 2 shows the TCS variations at the resonantfrequency of $11.02 \mathrm{GHz}$ as a function of the distance between the two coupled resonant structures, cases B and $\mathrm{C}$, respectively. The distances were measured between the centers of the two resonant apertures. The distances for the maximum and minimum TCSs for cases B and C are listed in Table 1. In terms of the relative arrangements of the magnetic dipole antenna elements, the distances for the maximum (minimum) TCSs were very close to the cases of halfwavelength dipole antennas $[33,34]$, where the maximum (minimum) gain ratios for two dipole antennas in collinear and side-by-side arrangements are achieved when the dipoles are approximately $1.0 \lambda(1.48 \lambda)$ and $0.7 \lambda(1.2 \lambda)$ away from each other, respectively. Here, the two terms collinear and side-by-side reflect the dipole arrangements [34]. Similarly, these two terms can also be applied to the equivalent magnetic current elements $\vec{M}$. As such, the collinear and
TABle 1: Center-to-center distance for maximum and minimum TCS cases (cases B and C).

\begin{tabular}{lcc}
\hline & Distance for case B & Distance for case C \\
\hline $\begin{array}{ll}\text { Maximum } \\
\text { TCS case }\end{array}$ & (i) $26.70 \mathrm{~mm}=0.981 \lambda$ & (ii) $19.50 \mathrm{~mm}=0.716 \lambda$ \\
$\begin{array}{l}\text { Minimum } \\
\text { TCS case }\end{array}$ & (iii) $38.55 \mathrm{~mm}=1.416 \lambda$ & (iv) $33.60 \mathrm{~mm}=1.234 \lambda$ \\
\hline
\end{tabular}

TABLE 2: Total TCS and TCS per unit aperture (at resonant frequency of $11.02 \mathrm{GHz}$ ).

\begin{tabular}{lccc}
\hline Case & $\begin{array}{c}\text { Total TCS } \\
\left(\mathrm{mm}^{2}\right)\end{array}$ & $\begin{array}{c}\text { TCS per unit } \\
\text { aperture }\left(\mathrm{mm}^{2}\right)\end{array}$ & $\begin{array}{c}\text { Distances for each } \\
\text { case }(\text { Table } 1)\end{array}$ \\
\hline $\mathrm{A}$ & 180.84 & 180.84 & - \\
$\mathrm{B}^{\max }$ & 395.08 & 197.54 & $(\mathrm{i})$ \\
$\mathrm{B}^{\min }$ & 348.32 & 174.16 & $($ iii) \\
$\mathrm{C}^{\max }$ & 533.35 & 266.68 & (ii) \\
$\mathrm{C}^{\min }$ & 305.15 & 152.58 & (iv) \\
$\mathrm{D}^{\max }$ & 1116.07 & 279.02 & (i) and (ii) \\
$\mathrm{D}^{\min }$ & 615.62 & 153.91 & (iii) and (iv) \\
$\mathrm{E}^{\max }$ & 341.72 & 170.86 & (i) and (ii) \\
$\mathrm{E}^{\min }$ & 380.25 & 190.13 & (iii) and (iv) \\
\hline
\end{tabular}

side-by-side cases correspond to cases B and C in Figure 1, respectively.

Table 2 shows the total TCS and TCS per unit aperture for cases $\mathrm{A}$ to $\mathrm{E}$ at the resonant frequency of $11.02 \mathrm{GHz}$. The superscripts "max" and "min" for each case represent the maximum and minimum TCS for cases B and C, respectively. For cases D and E, the distance for cases $\mathrm{B}$ and $\mathrm{C}$ was applied without sweeping the distance. The distances applied are summarized in the last column of Table 2. The TCSenhancing effect of case $\mathrm{C}^{\max }\left(\mathrm{C}^{\mathrm{min}}\right)$ was greater (smaller) than that of case $\mathrm{B}^{\max }\left(\mathrm{B}^{\mathrm{min}}\right)$, as seen in the third column of Table 2, implying that the mutual coupling in case $\mathrm{C}$ was 


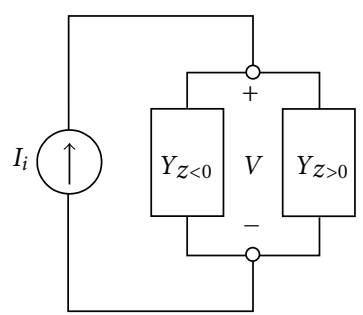

(a)

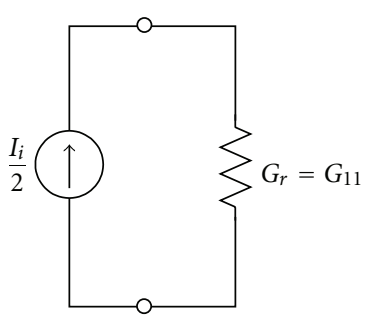

(b)
FIGURE 3: Equivalent circuit representation of single aperture for (a) general case and (b) its simplification from viewpoint of resonant transmission.

stronger than that in case B. This stronger coupling in the side-by-side case is very similar to the side-by-side case for dipole arrays. In addition, the resonant frequencies for cases $\mathrm{B}, \mathrm{C}$, and $\mathrm{D}$ remained the same as that for the single aperture case, that is, $11.02 \mathrm{GHz}$, as each aperture was resonant at the same frequency, and the distance between the two (or four) apertures did not produce any resonant frequency shift. However, the resonant frequency for case E did shift a little due to a slight difference between the resonant frequency for each aperture and that for the maximum TCS of the diagonal center-to-center distance [12]. The mutual coupling associated with the enhanced TCS will be further discussed later.

\section{Equivalent Circuit Representations with Self and Mutual Conductances}

3.1. Equivalent Circuit and Radiation Admittance for Single Aperture. Based on the numerical results in Table 2 and radiation conductance concept, an equivalent circuit was constructed. Figure 3(a) shows the equivalent circuit for a single aperture under general circumstances. The equivalent current source can be represented as $I_{i}=2 H_{0} \cdot l_{\text {eff }}[8]$, where $H_{0}$ is the incident magnetic field, and $l_{\text {eff }}$ is the effective physical length of $\vec{M}$, as marked in Figure 1. $Y_{z<0}$ and $Y_{z>0}$ are the total admittances for the half-space regions of $z<0$ and $z>0$, respectively, which become identical due to the symmetry of the structure. In general, each admittance in Figure 3(a) is expressed as $Y_{r}=G_{r}+j B_{r}$, where $G_{r}$ and $B_{r}$ are the radiating conductance and susceptance for the aperture, respectively. At the resonant frequency of 11.02 $\mathrm{GHz}$, the imaginary part $\left|B_{r}\right|$ became smaller than the real part $\left|G_{r}\right|$, as shown in Figure 4, implying that the most of the incident power was transmitted into the opposite region, while a small amount of power was stored near the aperture. Thus, a simplified equivalent circuit with only the conductance, as shown in Figure 3(b), was used to represent the resonant transmission to the $z>0$ region. In contrast, at an off-resonance, the transmitted power was significantly reduced, implying that the conductance was decreased and the susceptance became more dominant than the conductance. Indeed, this represents that the power was not transmitted to the opposite region but rather remained near the aperture. This was also observed from

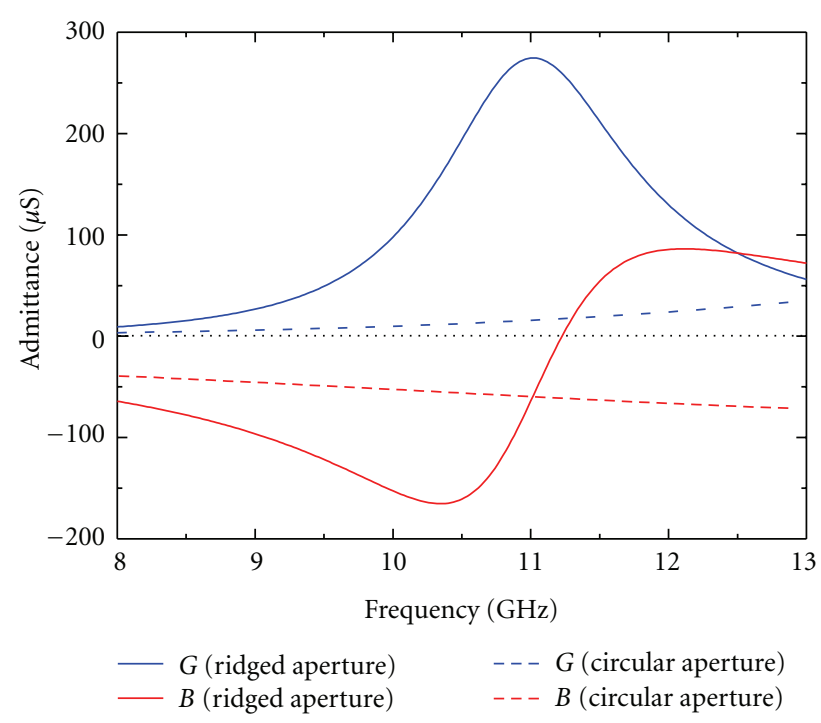

Figure 4: Admittance (conductance $G$ and susceptance $B$ ) for ridged circular aperture and original host circular aperture with diameter of $7.5 \mathrm{~mm}$.

the admittance (conductance and susceptance) at an offresonant frequency (i.e., except around $11.02 \mathrm{GHz}$ ) and the admittance over the whole frequency region for the original circular aperture without any ridges, shown in Figure 4. Derivations of the admittance for the ridged and original host circular apertures were conducted using the analytic expression in [35] and the 3D FDTD numerical results, as shown later in (4). Note that the discrepancy in the frequency points for the maximum radiation conductance and zero susceptance of the coupled subwavelength aperture was not that abnormal, since a small frequency difference has also been observed between the maximum radiation resistance and zero reactance of a dipole [34]. Thus, the duality of both types of structure allows them to be considered as related to each other, which will be discussed later. However, if the thickness of the structure is included in the analysis (as in [12]), this small frequency difference between the maximum radiation conductance and zero susceptance of the structure will not hold any more and could be larger than that of the present structure.

3.2. Self-Conductance and Effective Length of Magnetic Dipole Antenna Element. As shown in Figure 1, the magnetic dipole antenna element $\vec{M}$, which is the same as the equivalent magnetic current or virtual magnetic current in the previous section, yet expressed differently in this case since this terminology is more convenient for comparison with conventional dipole antennas, is considered to be virtually generated between the two ridges, since a strong electric field is induced in this region. As the length of $\vec{M}$ is very short compared to the wavelength of the resonant frequency, that is, $l_{\text {eff }} \ll \lambda$, this element is considered to be the same as the dual structure of a Hertzian dipole, for which the radiation resistance is given by $R_{r}=80 \pi^{2} \cdot\left(l_{\mathrm{H}} / \lambda\right)^{2}[36]$, where $l_{\mathrm{H}}$ is the physical length of the Hertzian dipole. Thus, 


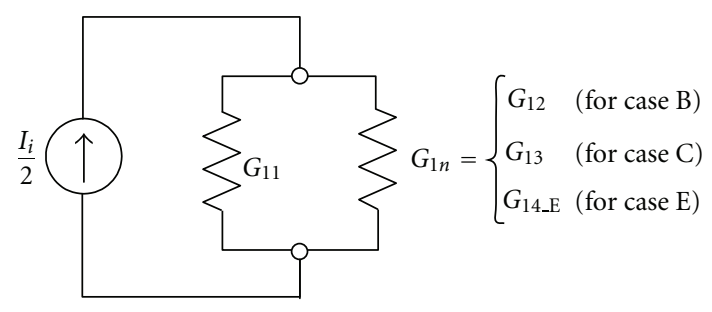

(a)

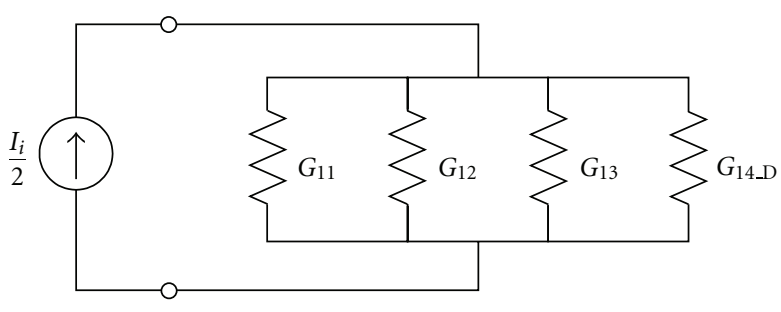

(b)

Figure 5: Equivalent circuit representation for (a) cases B, C, and E and (b) case D.

the radiation conductance of $\vec{M}$ for the ridged aperture is written as follows based on the duality of a Hertzian dipole,

$$
G_{r}=\frac{4}{\eta_{0}^{2}} R_{r}=\frac{8 \pi}{3 \eta_{0}}\left(\frac{l_{\mathrm{H}}}{\lambda}\right)^{2}
$$

which is already well known as Booker's relation [37]. This can also be referred to as the self-conductance of a single aperture, that is, $G_{11}=G_{r}$ (Figure 3(b)), together with $l_{\text {eff }}=l_{\mathrm{H}}$. The subscript 11 of $G_{11}$ represents not only the self-radiating conductance but also aperture 1 of case A in Figure 1. The transmitted power $P_{\text {trans }}$ for $G_{11}$ with the sourcing current $I_{i} / 2$, as shown in Figure 3(b), is then given as

$$
P_{\text {trans }}=\frac{\left|I_{i}\right|^{2}}{4 G_{11}}=\frac{\left|2 H_{0} \cdot l_{\text {eff }}\right|^{2}}{4 \cdot\left(8 \pi / 3 \eta_{0}\right)\left(l_{\text {eff }} / \lambda\right)^{2}}=\frac{1}{2} \eta_{0}\left|H_{0}\right|^{2} \cdot \frac{3 \lambda^{2}}{4 \pi} .
$$

If this transmitted power is then divided by the incident power density, that is, $P_{\text {inc }}=\eta_{0}\left|H_{0}\right|^{2} / 2$, the TCS is $3 \lambda^{2} /(4 \pi) \mathrm{m}^{2}$, which agrees well with the numerically obtained TCS for case A and is exactly the same as the maximum TCS for general 3D resonant apertures, as predicted by the coupled-mode theory [38]. From (2), $G_{11}$ with the proportional coefficient $g_{11}$ can be rewritten as $G_{11}=\left(g_{11} / \eta_{0}\right) \cdot\left(l_{\text {eff }} / \lambda\right)^{2}$, where $g_{11}=8 \pi / 3=8.220$. Meanwhile, $G_{11}$ can also be alternatively expressed as in [35], that is,

$$
G_{11}=\operatorname{Re}\{Y\}=\operatorname{Re}\left\{\frac{P^{*}}{|V|^{2}}\right\}=\operatorname{Re}\left[\frac{\left\{\left(\vec{E} \times \vec{H}^{*}\right) \cdot d \vec{S}\right\}^{*}}{|V|^{2}}\right],
$$

where $P$ is the radiated power from the aperture, $V$ is the voltage across the two ridges, as depicted in Figure 3(a), and $d \vec{S}$ is the surface vector normal to the aperture. $P^{*}$ and $|V|$ were easily obtained from the electromagnetic fields of the aperture at $z=0$ via the 3D FDTD simulations, from which the self-conductance was found to be $G_{11}=274.51 \mu \mathrm{S}$. From (4) and the previous relation of $G_{11}, l_{\text {eff }}=3.058 \mathrm{~mm}$ was also obtained, which was much shorter than the wavelength of the resonant frequency at $11.02 \mathrm{GHz}$, that is, $27.223 \mathrm{~mm}$, while slightly longer than the ridge width of $2.1 \mathrm{~mm}$ due to the strong confinement of the electric field in the gap between the two ridges with its fringing around the gap.
TABLE 3: Self and mutual conductances of coupled ridged circular apertures.

\begin{tabular}{lcc}
\hline \multicolumn{2}{c}{ Self and mutual conductances } & Cases \\
\hline$G_{11}$ & $274.51 \mu \mathrm{S}$ & $\mathrm{A}$ \\
$G_{12}^{\max }$ & $-23.18 \mu \mathrm{S}$ & $\mathrm{B}^{\max }$ \\
$G_{13}^{\max }$ & $-88.36 \mu \mathrm{S}$ & $\mathrm{C}^{\max }$ \\
$G_{14-\mathrm{D}}^{\max } / G_{14-\mathrm{E}}^{\max }$ & $14.94 / 16.08 \mu \mathrm{S}$ & $\mathrm{D}^{\max } / \mathrm{E}^{\max }$ \\
$G_{12}^{\min }$ & $10.50 \mu \mathrm{S}$ & $\mathrm{B}^{\min }$ \\
$G_{13}^{\min }$ & $50.79 \mu \mathrm{S}$ & $\mathrm{C}^{\min }$ \\
$G_{14 \_\mathrm{D}}^{\min } / G_{14 \_\mathrm{E}}^{\min }$ & $-13.31 /-13.38 \mu \mathrm{S}$ & $\mathrm{D}^{\min } / \mathrm{E}^{\min }$ \\
\hline
\end{tabular}

Consequently, the proposition to obtain self-conductance utilizing a Hertzian dipole is clearly convincing.

3.3. Equivalent Circuits and Mutual Conductances for Coupled Structures. For the maximum TCS in case B (case $\mathrm{B}^{\max }$ in Table 2), the mutual conductance $G_{12}^{\max }$ between apertures 1 and 2 together with $G_{11}$ were considered for a proper equivalent circuit representation. Similar to obtaining $G_{11}$, $G_{12}^{\max }$ was also simply expressed as $G_{12}^{\max }=\left(g_{12}^{\max } / \eta_{0}\right) \cdot\left(l_{\text {eff }} / \lambda\right)^{2}$, where $g_{12}^{\max }$ is the proportional coefficient for $G_{12}^{\max }$. As with the notation for the self-radiating conductance $G_{11}$, the subscript 12 of $G_{12}^{\max }$ represents the mutual coupling effect between apertures 1 and 2, as depicted in case B in Figure 1. Subscripts 13 and 14 hereinafter are also termed in the same way. The corresponding equivalent circuit was established by replacing $G_{11}$ in Figure 3(b) with a parallel connection of $G_{11}$ and $G_{12}^{\max }$, as shown in Figure 5(a). The total conductance for case $B^{\max }$ is then expressed as

$$
G_{\mathrm{B}}^{\max }=G_{11}+G_{12}^{\max }=\left(g_{11}+g_{12}^{\max }\right) \frac{1}{\eta_{0}}\left(\frac{l_{\mathrm{eff}}}{\lambda}\right)^{2} .
$$

Recalling the total TCS for case $\mathrm{B}^{\max }$ in Table 2, that is, $395.08 \mathrm{~mm}^{2}=0.533 \lambda^{2} \mathrm{~m}^{2}, G_{12}^{\max }=-23.18 \mu \mathrm{S}$ was obtained using $P_{\text {trans }}=\left|I_{i}\right|^{2} /\left(4 G_{\mathrm{B}}^{\max }\right)$ (similar to (3)) with (5). The mutual conductance between apertures 1 and 3 for case $C^{\max }$ was also obtained in the same way, that is, $G_{13}^{\max }=-88.36 \mu \mathrm{S}$ (Figure 5(a) also holds for this case). Similarly, the mutual conductance for cases $\mathrm{B}^{\mathrm{min}}$ and $\mathrm{C}^{\mathrm{min}}$ was obtained, and $G_{12}^{\mathrm{min}}$ and $G_{13}^{\min }$ are listed in Table 3, which summarizes the selfconductance $G_{11}$ and mutual conductances $G_{1 n}(n=2,3$, and 4) for each case. 
Meanwhile, the total conductance for case D (both maximum and minimum TCS cases are applied) is written as

$$
\begin{aligned}
G_{\mathrm{D}} & =G_{11}+G_{12}+G_{13}+G_{14} \\
& =\left(g_{11}+g_{12}+g_{13}+g_{14}\right) \frac{1}{\eta_{0}}\left(\frac{l_{\mathrm{eff}}}{\lambda}\right)^{2},
\end{aligned}
$$

where $g_{14}$ is also the proportional coefficient for the mutual conductance $G_{14}$. The parallel-in-echelon elements, that is, apertures 1 and 4 , and $G_{14}$ were also obtained, since $G_{11}, G_{12}$, and $G_{13}$ were already known. The corresponding equivalent circuit for case $\mathrm{D}$ was represented as shown in Figure 5(b). In a similar way to cases B and C, from the total TCS for case $\mathrm{D}^{\max }\left(\mathrm{D}^{\mathrm{min}}\right)$ in Table 2, $G_{14_{-} \mathrm{D}}^{\max }=14.94 \mu \mathrm{S}$ $\left(G_{14 D}^{\min }=-13.31 \mu \mathrm{S}\right)$ was obtained (see Table 3$)$, which was much smaller than $G_{12}$ and $G_{13}$, yet a different sign. In contrast, $G_{14}$ E was obtained from the TCS for case E in Table 2 and (5) using $g_{14}$ instead of $g_{12}^{\max }$, as listed in Table 3. Both $G_{14 \_D}^{\max }$ and $G_{14 \_\mathrm{E}}^{\max }$ (or $G_{14 \_D}^{\min }$ and $G_{14 \_\mathrm{E}}^{\mathrm{min}}$ ) were almost the same. Hence, the results confirmed the assumption of an equivalent magnetic current element $\vec{M}$ for subwavelength ridged circular apertures, and the very simple equivalent circuit representations were quite reasonable. Furthermore, the similar quantities for $G_{14 \_D}^{\max }$ and $G_{14 \_\mathrm{E}}^{\mathrm{max}}$ (or $G_{14_{-} \mathrm{D}}^{\mathrm{min}}$ and $G_{14 \_\mathrm{E}}^{\mathrm{min}}$ ) showed that the mutual conductance between two apertures can be obtained as long as the self-conductance of a single element and the total TCS for the two apertures are known, that is, without solving cases B to E totally and even when the two apertures are arbitrarily located.

3.4. Duality between Coupled Ridged Apertures and Multiple Dipole Antennas. From the previous procedures for obtaining the self-conductance and mutual conductances, the relations between the total conductances and the TCSs can be explained. Clearly, the summation of $G_{11}$ and $G_{12}$ for case B was larger than the summation of $G_{11}$ and $G_{13}$ for case C, while the TCS for case B was much lower than the TCS for case C. Thus, the TCS was found to be inversely proportional to the summation of the self and mutual conductances, as confirmed by the results in Tables 2 and 3. While this has already been discussed by the present authors according to the quantitative relations [12], it should be noted that a negative mutual conductance enhanced the TCS. As for a dual structure, the self and mutual resistances of half-wavelength dipole antenna arrays are listed in Table 4. Here too, the gain ratio of the structure is also inversely proportional to the summation of the self and mutual resistances [33], where the gain ratio was obtained as a function of the distance and arrangement of the two dipole elements, as with the present structures. More specifically, the gain ratio was maximized (minimized) when the mutual resistance was minimized (maximized) at a center-to-center distance of $1.0 \lambda(1.48 \lambda)$ and $0.7 \lambda(1.2 \lambda)$ in the collinear and side-by-side arrangements, respectively, and the relations were very similar to the distances satisfying the inverse proportionality between the TCSs and the summation of the conductances, as mentioned earlier. When extending
TABLE 4: Self and mutual resistances of half-wavelength dipole antennas.

\begin{tabular}{lcc}
\hline Self and mutual resistances & $\begin{array}{c}\text { Distance for each } \\
\text { case (Table 1) }\end{array}$ \\
\hline$R_{11}$ & $73.130 \Omega$ & - \\
$R_{12}^{\max }$ & $-4.173 \Omega$ & $($ i) \\
$R_{13}^{\max }$ & $-24.309 \Omega$ & (ii) \\
$R_{14}^{\max }$ & $8.822 \Omega$ & (i) \& (ii) \\
$R_{12}^{\min }$ & $1.573 \Omega$ & (iii) \\
$R_{13}^{\min }$ & $14.926 \Omega$ & (iv) \\
$R_{14}^{\min }$ & $-3.809 \Omega$ & (iii) \& (iv) \\
\hline
\end{tabular}

TABLE 5: Duality between half-wavelength dipole antenna array and ridged circular apertures.

\begin{tabular}{lc}
\hline Dipole antenna array [33] & $\begin{array}{c}\text { Coupled ridged circular apertures } \\
\text { (this work) }\end{array}$ \\
\hline Gain ratio & Total TCS \\
Self resistance & Self conductance \\
Mutual resistance & Mutual conductance \\
\hline
\end{tabular}

the inverse proportionalities, $G_{14}^{\max }\left(G_{14}^{\min }\right)$ had a minimal effect on the TCS in case $E^{\max }\left(E^{\text {min }}\right)$, as shown in the TCS per unit aperture column in Table 2 , in contrast to case $\mathrm{B}^{\max }$ or $\mathrm{C}^{\max }\left(\mathrm{B}^{\mathrm{min}}\right.$ or $\left.\mathrm{C}^{\mathrm{min}}\right)$. Plus, the small positive (negative) value of $G_{14}^{\max }\left(G_{14}^{\min }\right)$ was quite similar to the mutual resistance $R_{14}^{\max }\left(R_{14}^{\min }\right)$, shown in Table 4 , in the parallel-in-echelon case of a half-wavelength dipole antenna. After all, by comparing the self and mutual conductances with the self and mutual resistances as listed in Tables 3 and 4, the negative or positive signs of them are exactly the same, and the quantities' proportions among them are quite similar. Thus, despite the physical dissimilarities of the two resonant structures, the key physical quantities regarding the resonance behaviors interacting with incident electromagnetic waves were closely correlated due to the duality, as pointed out in Table 5.

\section{Conclusion}

The resonant power transmissions of various single and multiple subwavelength ridged circular aperture arrangements in a thin conducting screen were quantitatively investigated using equivalent circuits via the TCS with an admittance concept. An equivalent magnetic current element was introduced to effectively investigate the mutual coupling effect. As a result, for each case, the TCS for the ridged apertures was found to be inversely proportional to the summation of the conductances. Furthermore, the gain ratios in half-wavelength dipole antenna arrangements for both the maximum and minimum cases corresponded to the maximum and minimum TCSs for the current structures, respectively. Therefore, these observations identified the duality between coupled subwavelength ridged circular apertures and electric dipole antenna arrays. The current results may be useful for analyzing and/or synthesizing pairs of subwavelength metallic structures for such applications as 
optical data storage, high resolution near-field microscopy, and FSS.

\section{Acknowledgments}

The work of J.-E. Park and J.-W. Song was supported in part by the 2nd stage of Brain Korea 21 (BK21) at Kyungpook National University. The work of J.-E. Park was supported in part by the Korea Student Aid Foundation (KOSAF) Grant funded by the Korean government (MEST) (no. S2-2009000-00793-1).

\section{References}

[1] H. A. Bethe, "Theory of diffraction by small holes," Physical Review, vol. 66, pp. 163-182, 1944.

[2] A. Roberts, "Electromagnetic theory of diffraction by a circular aperture in a thick, perfectly conducting screen," Journal of the Optical Society of America A, vol. 4, no. 10, pp. 1970-1983, 1987.

[3] R. F. Harrington, "Resonant behavior of a small aperture backed by a conducting body," IEEE Transactions on Antennas and Propagation, vol. 30, no. 2, pp. 205-212, 1982.

[4] Y. Leviatan, "Electromagnetic coupling between two halfspace regions separated by two slot-perforated parallel conducting screens," IEEE Transactions on Microwave Theory and Techniques, vol. 36, no. 1, pp. 44-52, 1988.

[5] J.-I. Lee, J.-H. Ko, and Y.-K. Cho, "Electromagnetic transmission through slits in two adjacent conducting parallel-plates," The Journal of Korean Institute of Electromagnetic Engineering and Science, vol. 20, no. 1, pp. 83-90, 2009.

[6] Y. K. Cho, K. W. Kim, J. H. Ko, and J. I. Lee, "Transmission through a narrow slot in a thick conducting screen," IEEE Transactions on Antennas and Propagation, vol. 57, no. 3, pp. 813-816, 2009.

[7] J.-E. Park, J. Yeo, J.-I. Lee, J.-W. Ko, and Y.-K. Cho, "Resonant transmission of an electrically small aperture with a ridge," Journal of Electromagnetic Waves and Applications, vol. 23, no. 14-15, pp. 1981-1990, 2009.

[8] J. E. Park and Y. K. Cho, "Comparison of transmission resonance phenomena through small coupling apertures between two kinds of transmission resonance structures," in Proceedings of the 20th URSI International Symposium on Electromagnetic Theory (EMTS '10), pp. 744-747, Berlin, Germany, August 2010.

[9] J.-H. Ko, Y.-K. Cho, J. Yeo, and J.-I. Lee, "A Study on the resonant transmission through a ridge-loaded small circular aperture," The Journal of Korean Institute of Electromagnetic Engineering and Science, vol. 22, no. 6, pp. 654-660, 2011.

[10] J. Yeo, J. H. Ko, J. E. Park, J. I. Lee, and Y. K. Cho, "Analysis of resonant transmission characteristics of two sub-wavelength apertures with a ridge located in parallel," in Proceedings of the IEEE Antennas and Propagation on Symposium, Toronto, Canada, July 2010.

[11] J.-I. Lee, Y.-K. Cho, J.-H. Ko, and J. Yeo, "Resonant transmission through a pair of ridge-loaded circular sub-wavelength apertures," Progress In Electromagnetics Research M, vol. 24, pp. 113-126, 2012.

[12] J.-E. Park, K. Y. Kim, and J.-W. Song, "Resonant power transmission through coupled subwavelength ridged circular apertures," Journal of Electromagnetic Waves and Applications, vol. 26, no. 4, pp. 423-435, 2012.
[13] J.-H. Ko, J.-I. Lee, and Y.-K. Cho, "Electromagnetic scattering by a two-dimensional periodic array of small resonant apertures," The Journal of Korean Institute of Electromagnetic Engineering and Science, vol. 22, no. 3, pp. 320-326, 2011.

[14] T. W. Ebbesen, H. J. Lezec, H. F. Ghaemi, T. Thio, and P. A. Wolff, "Extraordinary optical transmission through subwavelength hole arrays," Nature, vol. 391, no. 6668, pp. 667669, 1998.

[15] E. X. Jin and X. Xu, "Finite-difference time-domain studies on optical transmission through planar nano-apertures in a metal film," Japanese Journal of Applied Physics, vol. 43, no. 1, pp. 407-417, 2004.

[16] K. Şendur, W. Challener, and C. Peng, "Ridge waveguide as a near field aperture for high density data storage," Journal of Applied Physics, vol. 96, no. 5, pp. 2743-2752, 2004.

[17] E. X. Jin and X. Xu, "Plasmonic effects in near-field optical transmission enhancement through a single bowtie-shaped aperture," Applied Physics B, vol. 84, no. 1-2, pp. 3-9, 2006.

[18] H. Guo, T. P. Meyrath, T. Zentgraf et al., "Optical resonances of bowtie slot antennas and their geometry and material dependence," Optics Express, vol. 16, no. 11, pp. 7756-7766, 2008.

[19] L. Wang and X. Xu, "High transmission nanoscale bowtieshaped aperture probe for near-field optical imaging," Applied Physics Letters, vol. 90, no. 26, Article ID 261105, 2007.

[20] T. Grosjean, M. Mivelle, F. I. Baida, G. W. Burr, and U. C. Fischer, "Diabolo nanoantenna for enhancing and confining the magnetic optical field," Nano Letters, vol. 11, no. 3, pp. 1009-1013, 2011.

[21] B. Lee, I. M. Lee, S. Kim, D. H. Oh, and L. Hesselink, "Review on subwavelength confinement of light with plasmonics," Journal of Modern Optics, vol. 57, no. 16, pp. 1479-1497, 2010.

[22] L. Wang, S. M. Uppuluri, E. X. Jin, and X. Xu, "Nanolithography using high transmission nanoscale bowtie apertures," Nano Letters, vol. 6, no. 3, pp. 361-364, 2006.

[23] A. Kinkhabwala, Z. Yu, S. Fan, Y. Avlasevich, K. Müllen, and W. E. Moerner, "Large single-molecule fluorescence enhancements produced by a bowtie nanoantenna," Nature Photonics, vol. 3, no. 11, pp. 654-657, 2009.

[24] S. Park and J. W. Hahn, "Plasmonic data storage medium with metallic nano-aperture array embedded in dielectric material," Optics Express, vol. 17, no. 22, pp. 20203-20210, 2009.

[25] H. G. Frey, S. Witt, K. Felderer, and R. Guckenberger, "Highresolution imaging of single fluorescent molecules with the optical near-field of a metal tip," Physical Review Letters, vol. 93, no. 20, pp. 1-200801, 2004.

[26] M. Mansuripur, A. R. Zakharian, A. Lesuffleur et al., "Plasmonic nano-structures for optical data storage," Optics Express, vol. 17, no. 16, pp. 14001-14014, 2009.

[27] P. Zijlstra, J. W. M. Chon, and M. Gu, "Five-dimensional optical recording mediated by surface plasmons in gold nanorods," Nature, vol. 459, no. 7245, pp. 410-413, 2009.

[28] Z. Rao, L. Hesselink, and J. S. Harris, "High transmission through ridge nano-apertures on vertical-cavity surfaceemitting lasers," Optics Express, vol. 15, no. 16, pp. 1042710438, 2007.

[29] C. E. Talley, J. B. Jackson, C. Oubre et al., "Surface-enhanced Raman scattering from individual $\mathrm{Au}$ nanoparticles and nanoparticle dimer substrates," Nano Letters, vol. 5, no. 8, pp. 1569-1574, 2005.

[30] L. Tang, D. A. B. Miller, A. K. Okyay et al., "C-shaped nanoaperture-enhanced germanium photodetector," Optics Letters, vol. 31, no. 10, pp. 1519-1521, 2006. 
[31] B. A. Munk, Finite Antenna Arrays and FSS, John Wiley \& Sons, Hoboken, NJ, USA, 2003.

[32] A. Taflove and S. C. Hagness, Computational Electrodynamics: The Finite-Difference Time-Domain Method, Artech House, Norwood, Mass, USA, 3rd edition, 2005.

[33] P. S. Carter, "Circuit relations in radiating systems and applications to antenna problems," Proceedings of the Institute of Radio Engineers, vol. 20, no. 6, pp. 1004-1041, 1932.

[34] C. A. Balanis, Antenna Theory, Wiley-Interscience, New Jersey, NJ, USA, 3rd edition, 2005.

[35] R. F. Harrington, Time-Harmonic Electromagnetic Fields, chapter 4, McGraw-Hill, New York, NY, USA, 1961.

[36] U. Inan and A. Inan, Electromagnetic Waves, Prentice Hall, New Jersey, NJ, USA, 2000.

[37] H. G. Booker, "Slot aerials and their relation to complementary wire aerials (Babinet's principle)," Journal of the Institution of Electrical Engineers A, vol. 93, no. 4, pp. 620-626, 1946.

[38] L. Verslegers, Z. Yu, P. B. Cartysse, and S. Fan, "Temporal coupled- mode theory for resonant apertures," Journal of the Optical Society of America B, vol. 27, no. 10, pp. 1947-1956, 2010. 

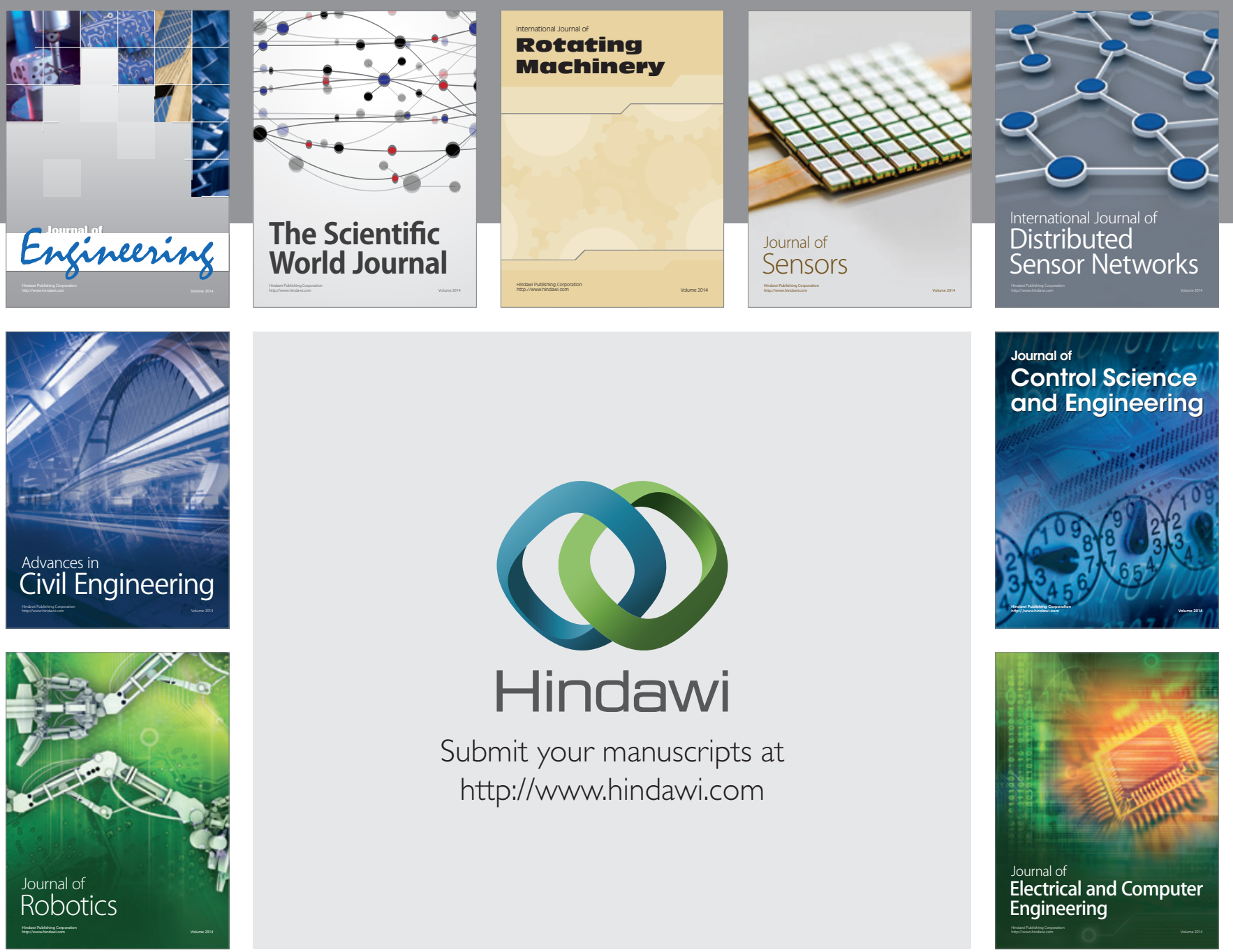

Submit your manuscripts at

http://www.hindawi.com
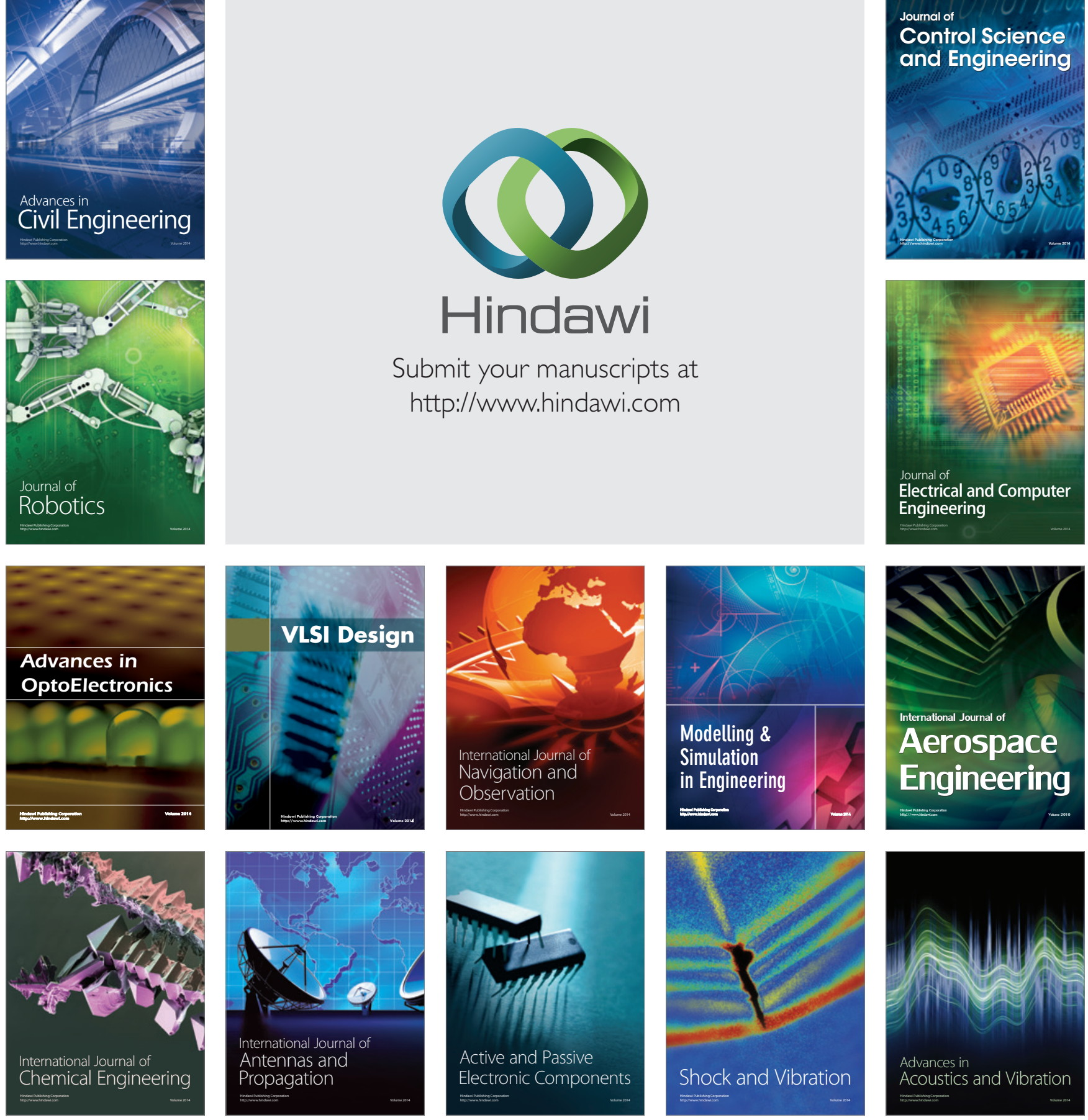